\title{
Acervos históricos del Herbario Nacional de México (MEXU): documental y bibliográfico
}

\author{
ARMANDO BUTANDA \\ Departamento de Botánica, Instituto de Biología, UNAM. Apdo. Postal 70-233, Delegación Coyoacán, \\ 04510, México, D.F.
}

\begin{abstract}
Resumen. Se reseñan los orígenes, las características y el contenido histórico del fondo documental y bibliográfico del Herbario Nacional de México (MEXU). Está integrado por 950 expedientes organizados en dos diferentes épocas y 1,548 libros de los siglos XVII a mediados del XX, provenientes en su mayor parte de las colecciones de las bibliotecas del Instituto Médico Nacional, el Museo Nacional de Historia Natural, la Comisión Geográfica Exploradora y la Dirección de Estudios Biológicos, instituciones desaparecidas que antecedieron al Instituto de Biología de la Universidad Nacional Autónoma de México.
\end{abstract}

Abstract. The origins, characteristics and historical contents of the documentary and bibliographic collections of the Herbario Nacional de México, are reviewed. At present the herbarium possesses 950 files organized in two periods and 1,548 books from the 17th to the middle 20th century, mostly of the library collections of the Instituto Médico Nacional, Museo Nacional de Historia Natural, Comisión Geográfica Exploradora and Dirección de Estudios Biológicos, precursors of the Instituto de Biología of the Universidad Nacional Autónoma de México.

\section{INTRODUCCIÓN}

Los acervos históricos son el testimonio de una institución científica que no surgió de la noche a la mañana, sino como resultado del trabajo de muchas generaciones de individuos que contribuyeron a su formación y conservación. Su consulta implica un acercamiento a un protagonista de cierta época o a un tema en estudio.

El Instituto de Biología, como depositario de colecciones nacionales de diversas especies vegetales y animales, y también de archivos y libros, se ha dado a la tarea de recuperar y mantener organizados los acervos que custodia, incluyendo los históricos: documentales y bibliográficos, heredados por la Dirección de Estudios Biológicos. Esta institución fue fundada en 1915, a partir de la fusión del Instituto Médico Nacional, el Museo de Historia Natural y la Comisión Geográfica Exploradora.

Los materiales que conforman el acervo histórico se encuentran en la Biblioteca del Herbario Nacional de México, en la sección denominada Acervo Histórico. La sección se divide en dos grupos: de documentos (que forma parte del Archivo Histórico de la Universidad Nacional Autónoma de México), registrado como Archivo Histórico del Instituto de Biología y el bibliográfico (registrado en la Dirección General de Bibliotecas, UNAM), como Acervo Histórico.

La información contenida en estos acervos es muy variada y de gran utilidad, no sólo para historiadores e investigadores botánicos, sino también para los de diferentes áreas de las ciencias. Su formación obedece a la necesidad de contar con una fuente de consulta fidedigna, que proporcione noticias que enriquezcan o cubran los espacios blancos de la historia de la biología o de la botánica, a veces desconocida o ignorada.

En vista de éstas y otras consideraciones, este trabajo tiene por objeto presentar una descripción global del contenido histórico y de las principales características de cada uno.

\section{ARCHIVO DE DOCUMENTOS}

El Archivo Histórico del Instituto de Biología, considerado dentro de los acervos históricos universitarios (UNAM) como uno de los de mayor antigüedad, junto con los de las Facultades de Medicina e Ingeniería, fue integrado a partir de 1985 por iniciativa de María Teresa Germán Ramírez (actual encargada). Ella se dio a la tarea de rescatar y catalogar los documentos adoptando las técnicas archivísticas del Centro de Estudios sobre la Universidad (CESU), que es la dependencia encargada del Archivo Histórico General de la UNAM. Se trata de documentos que, antes de esa fecha, aparentemente eran papeles viejos en un archivo muerto que esperaba en la biblioteca ser tirado al cesto de la basura, para dar espacio a documentos más recientes (Germán Ramírez, 1988). La búsqueda de información para conocer con exactitud el origen, la adquisición o estructuración de varias de las colecciones de plantas, libros, manuscritos, dibujos y pinturas que existen en el Instituto de Biología, llevó a María Teresa Germán a esos papeles viejos que afortunadamente, con labor curatorial, fueron salvados de la basura (Germán Ramírez, 1988).

En 1987, el archivo estaba formado por 700 expedientes (actualmente cuenta con 950) organizados en dos diferentes épocas o fondos; una mitad corresponde al Ins- 
tituto Médico Nacional 1888-1915, que estuvo instalado en el edificio de la calle de Balderas 94, y la otra mitad corresponde al Instituto de Biología 1930-1968, ubicado en sus inicios en la Casa del Lago en Chapultepec y luego en sus actuales instalaciones en Ciudad Universitaria (Galicia Miranda y Germán Ramírez, 1987).

Cada expediente contiene un número variable de documentos con información de investigadores o investigaciones vinculadas con el desarrollo del país o del Instituto de Biología, y del Herbario Nacional en diferentes épocas. Están clasificados y ordenados en ramos, subramos y series documentales.

Ejemplo de documentos valiosos son los que forman la correspondencia de los doctores Fernando Altamirano y Alfonso L. Herrera sobre terapéutica de diversas especies vegetales. Están también los del Subramo Proyectos, del Fondo Instituto Médico Nacional, organizado en tres series: Botánica, Zoología y Análisis químicos. Aquí se reúnen testimonios, en su mayoría manuscritos, que revelan los estudios realizados acerca de las plantas, animales y minerales, de su utilidad, aplicación y enfermedades que provocaban, los informes de los investigadores respectivos, información acerca de los colaboradores que enviaron las muestras, sitios de procedencia de éstas y aspectos médicos, químicos y clínicos y dosificaciones de prueba. Los documentos del Fondo Instituto de Biología, tienen también tres series: Botánica, Zoología y Químicos biológicos. De éstas, la más amplia es la de botánica, con 45 expedientes que contienen informes acerca de expediciones, solicitudes para estudios taxonómicos de plantas, los propios estudios y sus reportes, listas de plantas proporcionadas, permisos para colectores extranjeros y para exportar plantas, envíos de muestras de semillas, etcétera.

\section{COLECCIONES ESPECIALES}

En sección separada se localizan otras colecciones especiales, como el archivo de libretas de colectores (por ejemplo, de Faustino Miranda, Eizi Matuda, José Sánchez y Pedro Tenorio, entre otros). Hay también manuscritos de obras publicadas o inéditas de personal del Instituto de Biología o de asociados a éste; dibujos de plantas a lápiz y en color, algunas tomadas de obras conocidas como la de Cavanilles, Icones ét descriptiones plantarum o la de Sessé y Mociño, Flora Mexicana, elaborados por el dibujante Adolfo Tenorio, y acuarelas realizadas por pintores de la talla de José María Velasco o Adrián Unzueta para el Herbario Nacional, que en esa época formaba parte del Museo Nacional de México, 1900-1902.

Además existe un archivo fotográfico que está integrado por colecciones de positivos de muy diversas especies y lugares; negativos de ejemplares Tipo tomados en herbarios diferentes de Estados Unidos de América y de Gran Bretaña; diapositivas de plantas donadas por colectores como José Vázquez (de la flora de Morelos) y Hubert Kruse (de la flora de Guerrero).

\section{ACERVO BIBLIOGRÁFICO}

La integración del que actualmente se conoce dentro del Sistema Bibliotecario del Instituto de Biología como Acervo
Histórico del Herbario Nacional, se inició a fines de 1989, mediante un convenio del Departamento de Botánica del IBUNAM y la entonces Unidad de Bibliotecas de la Coordinación de la Investigación Científica. Durante 1990 y 1991 se llevó a cabo su formación seleccionando y separando de los estantes de la Biblioteca diversas obras para su inventario, clasificación, catalogación y organización.

El acervo se originó con material que abarcaba colecciones del siglo XVII hasta fines del XIX. Este material provenía de las bibliotecas del Instituto Médico Nacional, del Museo de Historia Natural, de la Comisión Geográfica Exploradora y de la Dirección de Estudios Biológicos, todas instituciones antecesoras del Instituto de Biología. El acervo, que está incluido en la base de datos LIBRUNAM, integra 787 títulos en 1,548 volúmenes, con un número aproximado de 4,722 tarjetas que conforman los catálogos para consulta.

Existe poca bibliografía sobre el contenido de este valioso acervo, por lo que la mayor parte de la información que se presenta ha sido obtenida del estudio y la revisión de la colección a lo largo de cinco años; sin embargo, en los trabajos de Dávila Aranda y Germán Ramírez (1991) y García Alcántara (1991) se pueden encontrar aspectos generales de ese contenido.

Cada obra existente en el Acervo Histórico tiene gran importancia para la historia de las ciencias, la taxonomía vegetal, la florística regional o en el desarrollo de las colecciones de la institución, y ante la imposibilidad, de describirlas con detalle en este artículo, nos limitaremos a enlistar algunas de ellas (Cuadro 1) que han sido reseñadas por Langman (1964) y Stafleu y Cowan (1976-1988).

\section{COMENTARIOS FINALES}

La breve relación presentada aquí sobre el contenido de estos dos acervos históricos, proporciona al lector elementos suficientes para valorar su importancia como auxiliares en investigación.

Los documentos representan asuntos que en otro tiempo tuvieron activo movimiento, y en el presente, como expedientes de archivo, siguen dando servicio como fuente histórica. Los libros, históricos o no, son fuente de información obligada en el inicio de cualquier estudio.

Creemos que este tipo de materiales son una herramienta y un recurso en la investigación; su consulta debe ser considerada como una alternativa en el estudio de las perspectivas de las ciencias vegetales.

\section{AGRADECIMIENTOS}

Agradecemos a la bióloga María Teresa Germán Ramírez las sugerencias, su revisión crítica y el habernos facilitado datos inéditos sobre el Archivo Documental, así como a los maestros Beatriz Gómez Varela y Antonio Bolívar Goyanes la correción de estilo del manuscrito final. 
Cuadro 1. Listado de títulos de obras por el siglo de edición

Siglo XVII

1635. "Historia Naturae" de Johannes Nieremberg, contiene muchas referencias a plantas mexicanas, y es el primer trabajo que utiliza las ilustraciones del manuscrito original del Protomédico de Indias Francisco Hernández.

1649. "Rerum Medicarum Novae Hispaniae Thesaurus sev Plantarum Animalium Mexicanorum" de Francisco Hernández, conocida como edición romana de Antonio Nardo Reccho.

1671. "Pinax Theatri Botanici" de Kaspar Bauhin, en la que se enlistan todas las plantas conocidas por el autor.

1671. "Prodomus Theatri Botanici" de Kaspar Bauhin, en la que el autor incluye la ilustración de cada planta descrita.

1678. "Hortus Indicus Malabaricus" de Hendrik Adriaan van Rheede tot Drakestein, obra sobre las plantas de la India que incluye las especies medicinales y los recursos de la flora de Malabar.

Siglo XVIII

1700. "Institutiones rei Herbariae" de Joseph Pitton De Tournefort, obra para el acervo de la Real Expedición Botánica.

1703. "Methodus Plantarum Emendata et Aucta" de Joanni Raji.

1714. "Journal des Observationes Physiques, Mathématiques et Botaniques" de Louis Feuiville.

1718. "Botanographia" de Caspar Commelin.

1741. "Historiae Naturalis" de Cayo Plinio Segundo (Plinio el Viejo), obra traducida y comentada por Francisco Hernández.

1755. "Plantarum Americanum" de Charles Plumier, editada por Joannes Burmannus.

1762. "Flora Española, o Historia de las Plantas, que se crían en España" de Joseph Quer.

1768. "Historia Muscorum" de Johann Jakob Dillenius.

1772. "Física de los árboles en la cual se trata de la Anatomía de las Plantas" de Henri Louis Duhamel du Monceau, traducida con varias notas por Casimiro Gómez Ortega.

1780. "Storia Antica del Messico" de Francisco Javier Clavijero, escrita originalmente en italiano; se ha traducido al español, inglés y alemán.

1781. "Icones Plantarum Rariorum" de Nicolao Josepho Jacquin.

1781. "Reliquiae Houstounianae" de William Houstoun, incluye la descripción de plantas colectadas por el autor en 1829 en el estado de Veracruz.

1784. "Parte Práctica de Linneo" de Carl von Linné, traducida por Antonio Paláu y Verdéra. Fue texto obligatorio de la Primera Cátedra de Botánica en México (1788) dictada por el botánico español Vicente Cervantes, quien urgió al director del Jardín Botánico de Madrid para que le enviara unos 30 ejemplares a la Nueva España para cada uno de sus alumnos.

1786. "Fundamentorum Botanicorum" de Carl von Linné: pars prima-secunda.

1790. "Opera Cum Edita, Tum Inedita" de Francisco Hernández, conocida como edición matritense, traducida por Casimiro Gómez Ortega.

1791. "Icones et Descriptiones Plantarum" de Antonio José Cavanilles; incluye varias plantas mexicanas.

1791. "Tableu Encyclopédique et Méthodique des Tois Règnes de la Nature" de Le Chevalier de la Mark.

1792. "Botanicorum Principis, Philosophia Botanica, Annotationibus" de Carl von Linne.

1799. "Plantarum Suculentarum Historia" de Agustín Pyramus De Candolle.

1799. "Species Plantarum" de Carol von Linné.

Siglo XIX

1801. "Lexicon Botanicum" de Joannis Beckmanni.

1802. "Descripción de las Plantas que D. Antonio Josef Cavanilles demostró en las Lecciones Públicas del año 1801" precedido de los principios elementales de la botánica.

1803. "Flora Boreali-Americana: sistems caracteres plantarum quas in America Septentrionali Collegit et Detexit", de Andre Michaux. 
1804. " Recherches Chiniques sur la Vegetation" de Theodore De Saussure.

1806. "Synopsis Plantarum in Flora Gallica Descriptarum" de Jean Baptiste Lamarck y A.P. Candolle.

1807. "The Botanic Garden: a poem in two parts with philosophical notes" de Erasmus Darwin.

1810. "Lichenographia Universalis" de Erik Acharius.

1813. "Théorie Éleméntaire de la Botanique" de Agustín Pyramus de Candolle.

1815. "Nova Genera Species Plantarum" de Alexandre De Humbolt y Aime Jacques Alexandre Bonpland, editada por Carol Sigismund Kunth.

1824. "Novorum Vegetabilium Descriptiones" De La Llave y Lexarza, en donde fueron descritas 60 especies mexicanas nuevas para la ciencia, y en la que los autores bautizan a los nuevos géneros con los nombres de algunos caudillos de la Independencia. Éstos son Aldama, Galeana, Hidalgoa, Morelosia, Roldana, Truianoa, entre otros.

1827. "Organographie Végétale ou Description Raisonné de Organes des Plantes" de Agustín Pyramus de Candolle.

1830. "Reliquiae Haenkenae" de Thadaeus Haenke

1836. "Materia Farmacéutica Vegetal o Botánica Médica" de José Oriol Ronquillo.

1837. "The Orchidaceae of Mexico and Guatemala" de Jeorge Bateman.

1839. "Plantas Hartwegianas" de George Bentham.

1840. "Nomenclator Botanicus" de Ernesto Theoph Steudel.

1846. "Nuevo Curso Elemental de Botánica" de Pío Bustamante y Rocha, escrito en el orden que se enseñó este ramo en el Jardín del Palacio Nacional.

1852. "The Botany of the Voyage of H. M. S. Herald" de Berthold Seemann.

1857. "Report on the United States and Mexican Boundary Survey" de William Hemsley Emory. Las ilustraciones dan una excelente idea del aspecto general de región.

1858. "Theoria Systematis Plantarum: accedit familiarum phanerogamarum..." de Jacobo Georgio Agardh.

1865. "Le Guide du Botaniste Herborisant" de Bernard Verlot.

1865. "Memoria de los Trabajos Ejecutados por la Comisión Científica de Pachuca en el año de 1864" de Ramón Almaraz

1867. "Genera Plantarum" de George Bentham y J. D. Hooker.

1872. "Traité des Plantes Médicinales Indigenes" de Antonin Bossu.

1873. "La Flore Mexicaine aux Environs de Córdova" de Omer de Malzine.

1876. "Darwiniana: essays and reviews pertaining to Darwinism" de Asa Gray.

1876. "Dictionnaire de Botanique" de M. H. Baillon.

1877. "Des Effets de la Fecondation Croisse et de la Fecondation Directe dans le Regne Vegetale" de Charles Robert Darwin.

1879. "Biología Centrali-Americana; ... Botany" de W. Botting Hemsley.

1886. "Historia de la Medicina: desde la época de los indios hasta la presente" de Francisco A. Flores.

1886. "Guide Éleméntaire D’Herborisations et de Botanique Practique" de Henry Ernest Baillon.

1887. "Flora Mexicana" de Martín Sessé y José Mariano Mociño.

1888. "La Viet et la Correspondence de Charles Darwin avec un chapitre autobiographique", publiés par son fils M. Francis Darwin, traduit de l'anglais par Henry C. de Varingny.

1888. "Un Discurso y un Catálogo de Plantas Clasificadas" de José Eleuterio González.

1888. "Cuatro Libros de la Naturaleza y Virtudes de las Plantas y Animales, de Uso Medicinal en la Nueva España" de Francisco Ximenez, reimpresiones de Antonio Peñafiel y Nicolás León.

1889. "Ensayo a la Materia Médica Vegetal de México" de Vicente Cervantes.

1889. "Memoria para una Bibliografía Científica de México en el Siglo XIX" de Manuel Olaguíbel; es el primer trabajo bibliográfico sobre la Flora de México.

1891. "Flora y Fauna del Estado Libre y Soberano de Oaxaca" de Manuel Martínez Gracida.

1893. "Plantae Novae Hispaniae" de Martín Sessé y José Mariano Mociño.

1893. "Consejos Prácticos para Colectar Plantas" de Lester Frank Ward, traducido del inglés por Fernando Altamirano.

1895. "Flora Sinóptica Mexicana" de Cassiano Conzatti y Lucio C. Smith.

1897. "Catálogo de Plantas Mexicanas (fanerógamas)" de Manuel Urbina.

1899. "Apuntes para la Flora Zacatecana" de Elías Amador.

1899. "Viaje de un Naturalista Alrededor del Mundo" de Charles Robert Darwin.

1899. "La Vegetación de México: recopilación y análisis de las principales clasificaciones propuestas" de José Ramírez 
Cuadro 1. Continuación.

Siglo XX (1900-1946)

1900. "La Descendencia del Hombre y la Selección en sus Relaciones con el Sexo" de Charles Robert Darwin.

1900. "La Vie des Plantes" de Paul Constantine.

1900. "Eléments de Paléobotanique" de R. Zeiller.

1902. "Nombres Geográficos Mexicanos del Estado de Veracruz" de Cecilio A. Robelo.

1902. "Sinonimia Vulgar y Científica de las Plantas Mexicanas" de José Ramírez.

1903. "Los Géneros Vegetales Mexicanos" de Cassiano Conzatti.

1904. "Estudios de Historia Natural" de José Ramírez.

1906. "Catálogo de Consulta de los Géneros y Subgéneros de que consta el Herbario del Instituto Médico Nacional" de Gabriel V. Alcocer.

1907. "Linnéporträtt" de Tycho Tullberg.

1909. "Ligeros Apuntes sobre la Flora del Estado de Sinaloa" de Ramón Ponce de León.

1914. "La Vegetación en los alrededores de la Capital de México" de Karl Friedrich Reiche.

1915. "Die Agaven: beitrage zu einer monographie" de von Alwin Berger.

1920. "El Estado de Oaxaca y sus Recursos Naturales" de Cassiano Conzatti.

1917. "Botanisches Lesebuch" de Karl Friedrich Reiche.

1924. "Botánica" de Alfonso L. Herrera.

1926. "Flora Excursoria en el Valle Central de México" de Karl Friedrich Reiche.

1928. "Lecturas Biológicas: una introducción a la ecología de los organismos" de Karl Friedrich Reiche.

1933. "Botánica General Organostática" de Guillermo Gándara.

1933. "Códices Indígenas de Algunos Pueblos del Marquesado del Valle de Oaxaca" publicados por el Archivo General de la Nación.

1933. "La Flóra del Estado de Jalisco" de Alfonso Manuel Castañeda.

1937. "Guía para el Curso de Botánica" de Maximino Martínez.

1937. "Catálogo de Nombres Vulgares y Científicos de Plantas Mexicanas" de Maximino Martínez.

1940. "The Badianus Manuscript (Codex Barberini, Latin 241) Vatican Library. An Aztec Herbal of 1552", traducción y anotaciones de Emily Walcott Emmart, quien tardó ocho años en la preparación de la edición facsimilar a la que realmente se debe la difusión del manuscrito de Martín de la Cruz traducido por Juan Badiano.

1942. "Historia de las Plantas de Nueva España" de Francisco Hernández.

1945. "Apuntes Relativos a la Flora de Yucatán" de Narciso Souza Novelo.

1946: " Flora Taxonómica Mexicana (plantas vasculares)" de Cassiano Conzatti.

\section{AGRADECIMIENTOS}

Agradecemos a la bióloga María Teresa Germán Ramírez las sugerencias, su revisión crítica y el habernos facilitado datos inéditos sobre el Archivo Documental, así como a los maestros Beatriz Gómez Varela y Antonio Bolívar Goyanes la correción de estilo del manuscrito final.

\section{LITERATURA CITADA}

Dávila Aranda PD, Germán Ramírez MT. 1991. Colecciones biológicas del Instituto de Biología: Herbario Nacional de México. UNAM, México.

Galicia Miranda V, Germán Ramírez MT. 1987. El acervo histórico en el Herbario Nacional de México. Resúmenes X Congreso Mexicano de Botánica, Guadalajara, Jal. Trabajo 466.

García Alcántara R. 1991. Historia de la Biblioteca del Herbario Nacional ("MEXU") y sus publicaciones. Tesina Colegio de Bibliotecología, Facultad de Filosofía y Letras, UNAM, México.

Germán Ramírez MT 1988. El Archivo Histórico del Instituto de Biología, un recurso en la investigación. En: Los acervos documentales de la UNAM (coloquio). UNAM, México, 113116 (Cuadernos del Archivo Histórico de la UNAM).

Langman IK. 1964. A selected guide to the literature on the flowering plants of Mexico. University of Pennsylvania Press, Philadelphia.

Stafleu FA, Cowan RS. 1976-1988. Taxonomic literature: a selective guide to botanical publications and collections with dates, commentaries and types. 2a. ed. Bohn, Scheltema \& Holkema. Utrech. $7 \mathrm{v}$. 